\title{
On a Generalized Burr Life-Testing Model: Characterization, Reliability, Simulation, and Akaike Information Criterion
}

\author{
M. Ahsanullah ${ }^{1}$, M. Shakil ${ }^{2, \star}$, B. M. Golam Kibria ${ }^{3}$, M. Elgarhy ${ }^{4}$
}

${ }^{1}$ Rider University, New Jersey, USA

${ }^{2}$ Miami Dade College, Hialeah, Florida, USA

${ }^{3}$ Florida International University, Florida, USA

${ }^{4}$ University of Jeddah, Jeddah, KSA

\section{ARTICLE INFO}

Article History

Received 22 Oct 2018

Accepted 22 Mar 2019

Keywords

Akaike information criterion

Characterization

Reliability

Truncated moment

\section{ABSTRACT}

For a continuous random variable X, M. Shakil, B.M.G. Kibria, J. Stat. Theory Appl. 9 (2010), 255-282, introduced a generalized Burr increasing, decreasing, and upside-down bathtub failure rate life-testing model. In this paper, we provide some characterizations of this life-testing model by truncated first moment, order statistics and upper record values. We also investigate the reliability, simulation, and Akaike information criterion for this model.

(c) 2019 The Authors. Published by Atlantis Press SARL. This is an open access article distributed under the CC BY-NC 4.0 license (http://creativecommons.org/licenses/by-nc/4.0/).

\section{INTRODUCTION}

For a continuous positive random variable $X$, the following increasing, decreasing, and upside-down bathtub failure rate life testing fiveparameter family of continuous probability density function (pdf) in terms of beta function has been introduced by Shakil and Kibria [1]:

$$
f_{X}(x)=\frac{p(\alpha)^{\nu-\frac{\mu}{p}}(\beta)^{\frac{\mu}{p}} x^{\mu-1}}{\mathrm{~B}\left(\frac{\mu}{p}, \nu-\frac{\mu}{p}\right)\left(\alpha+\beta x^{p}\right)^{\nu}},
$$

where $x>0, \alpha>0, \beta>0, \mu>0, \nu>0, p>0$, and where, in view of the definition of beta function, the parameters are chosen as such that $\nu>\frac{\mu}{p}$. For details, the interested readers are referred to Shakil and Kibria [1], and references therein. The cumulative distribution function (cdf) is given by

$$
\begin{gathered}
F_{X}(x)=\operatorname{Pr}(X \leq x)=\frac{p\left(\frac{\beta}{\alpha}\right)^{\frac{\mu}{p}}}{\mathrm{~B}\left(\frac{\mu}{p}, \nu-\frac{\mu}{p}\right)} \int_{0}^{x} \frac{t^{\mu-1}}{\left(1+\frac{\beta}{\alpha} t^{p}\right)^{\nu}} d t, \\
=\frac{p\left(\frac{\beta}{\alpha}\right)^{\frac{\mu}{p}}}{\mathrm{~B}\left(\frac{\mu}{p}, \nu-\frac{\mu}{p}\right)} \sum_{k=0}^{\infty}\left\{\frac{(-1)^{k} \Gamma(\nu+k)}{\Gamma(\nu)} \frac{\left(\frac{\beta}{\alpha}\right)^{k} x^{(\mu+p k)}}{(k !)(\mu+p k)}\right\},
\end{gathered}
$$




$$
\begin{gathered}
=\frac{p\left(\frac{\beta}{\alpha}\right)^{\frac{\mu}{p}}}{\mathrm{~B}\left(\frac{\mu}{p}, \nu-\frac{\mu}{p}\right)} \sum_{k=0}^{\infty}\left\{\frac{(-1)^{k}\left(\frac{\beta}{\alpha}\right)^{k}(\nu)_{k} x^{(\mu+p k)}}{(k !)(\mu+p k)}\right\} . \\
=\frac{\frac{p}{\mu}\left(\frac{\beta}{\alpha}\right)^{\frac{\mu}{p}} x^{\mu}}{\mathrm{B}\left(\frac{\mu}{p}, \nu-\frac{\mu}{p}\right)}{ }_{2} F_{1}\left(\nu, \frac{\mu}{p} ; \frac{\mu}{p}+1 ; \frac{-\beta}{\alpha} x^{p}\right),
\end{gathered}
$$

where ${ }_{2} F_{1}($.$) and (\nu)_{k}$ denote the Gauss hypergeometric and Pochhammer functions.

The $k$ th moment, $\alpha_{k}$, for some integer $k>0$, is given by

$$
\alpha_{k}=E\left(X^{k}\right)=\int_{0}^{\infty} x^{k} f_{X}(x) d x=\left(\frac{\alpha}{\beta}\right)^{\frac{k}{p}} \frac{\mathrm{B}\left(\frac{\mu+k}{p}, \nu-\frac{\mu+k}{p}\right)}{\mathrm{B}\left(\frac{\mu}{p}, \nu-\frac{\mu}{p}\right)}
$$

The first moment, when $k=1$ in (6), is given by

$$
\alpha_{1}=E(X)=\left(\frac{\alpha}{\beta}\right)^{\frac{1}{p}} \frac{\mathrm{B}\left(\frac{\mu+1}{p}, \nu-\frac{\mu+1}{p}\right)}{\mathrm{B}\left(\frac{\mu}{p}, \nu-\frac{\mu}{p}\right)},
$$

which is obviously a finite and fixed number for $\alpha>0, \beta>0, \mu>0, \nu>0, p>0$, and $\nu p>\mu$. The possible shapes of the pdf (1) are provided for some selected values of the parameters in Fig. 1 (a and b) below.

The effects of the parameters can easily be seen from the above graphs. From these graphs, it is observed that the above-said distribution is right skewed. Also, since the mode is the value of $x$ for which the probability density function $f_{X}(x)$ defined in $(1)$ is maximum, therefore differentiating (1), we have

$$
\frac{d f_{X}(x)}{d x}=\frac{\left(\alpha(\mu-1)-\beta(\nu p-\mu+1) x^{p}\right) f_{X}(x)}{x\left(\beta x^{p}+\alpha\right)},
$$

(a)

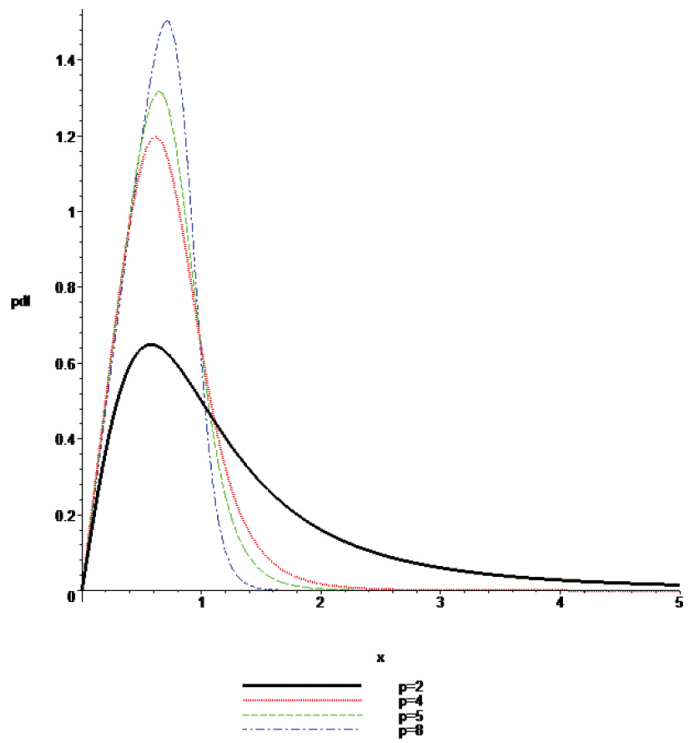

(b)

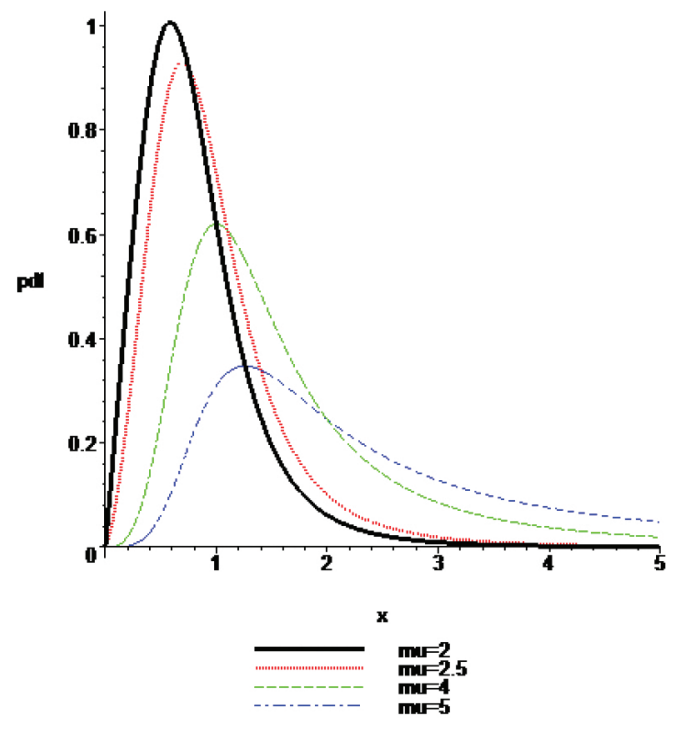

Figure 1 PDF Plots for: (a) $\alpha=1, \beta=1, v=2, \mu=2, p=2,4,5,8$ (left); and (b) $\alpha=1, \beta=1, v=2, p=3, \mu=2,2.5,4,5$ (right). 
which, when equated to 0 , gives the mode of the $\operatorname{pdf}(1)$ as

$$
x=\left[\frac{\alpha(\mu-1)}{\beta(\nu p-\mu+1)}\right]^{\frac{1}{p}}=\delta(\text { say }), \alpha>0, \beta>0, \mu>0, \nu>0, p>0,
$$

which exists provided $\mu>1$, and $\nu p>\mu$. Differentiating (8) and using (9), it can easily be seen by simple arguments that $\frac{d^{2} f_{X}(\delta)}{d x^{2}}<0$. Thus the maximum value of the probability density function (1) is given by $f_{X}(\delta)$, where $\delta$ is given by (9). It follows that the probability density function (1) is unimodal.

The paper is organized as follows. In Section 2, we present some characterization results. We provide reliability results in Section 3. The Akaike information criterion (AIC), etc., are discussed in Section 4. Finally, some concluding remarks are presented in Section 5.

\section{CHARACTERIZATION RESULTS}

Since the truncated distributions arise in practical statistics where the ability of record observations is limited to a given threshold or within a specified range, there has been a great interest, in recent years, in the characterizations of probability distributions by truncated moments. The development of the general theory of the characterizations of probability distributions by truncated moment began with the work of Galambos and Kotz [2], Kotz and Shanbhag [3], Glänzel et al. [4], and Glänzel [5]. For recent developments, we refer our readers to Ahsanullah [6], and references therein. In this section, we provide the proposed characterizations of the said distribution by truncated moment, order statistics, and upper record values. For this, we shall need the following assumption and lemmas.

\subsection{Assumption and Lemmas}

Assumption 2.1.1. Suppose the random variable $X$ is absolutely continuous with the cumulative distribution function $F(x)$ and the probability density function $f(x)$. We assume that $\gamma=\inf \{x \mid F(x)>0\}$, and $\delta=\sup \{x \mid F(x)<1\}$. We also assume that $f(x)$ is a differentiable for all $x$, and $E(X)$ exists.

Lemma 2.1.1. Under the Assumption 2.1.1, if $E(X \mid X \leq x)=g(x) \tau(x)$, where $\tau(x)=\frac{f(x)}{F(x)}$ and $g(x)$ is a continuous differentiable function of $x$ with the condition that $\int_{\gamma}^{x} \frac{u-g^{\prime}(u)}{g(u)} d u$ is finite for all $x, \gamma<x<\delta$, then $f(x)=c e^{\int_{\gamma}^{x} \frac{u-g^{\prime}(u)}{g(u)} d u}$, where $c$ is determined by the condition $\int_{\gamma}^{\delta} f(x) d x=1$

Proof: See Ahsanullah and Shakil [7].

Lemma 2.1.2. Under the Assumption 2.1.1, if $E(X \mid X \geq x)=g(x) r(x)$, where $r(x)=\frac{f(x)}{1-F(x)}$ and $g(x)$ is a continuous differentiable function of $x$ with the condition that $\int_{x}^{\delta} \frac{u+g^{\prime}(u)}{g(u)} d u$ is finite for all $x, \gamma<x<\delta$, then $f(x)=c e^{-\int_{x}^{\delta} \frac{u+g^{\prime}(u)}{g(u)} d u}$, where $c$ is determined by the condition $\int_{\gamma}^{\delta} f(x) d x=1$.

Proof: See Ahsanullah and Shakil [7].

\subsection{Characterization by Truncated First Moment}

We provide the proposed characterizations in Theorems 2.2.1 and 2.2.2.

Theorem 2.2.1. If the random variable $X$ satisfies the Assumption 2.1.1, with $\gamma=0$ and $\delta=\infty$, then $E(X \mid X \leq x)=g(x) \frac{f(x)}{F(x)}$, where

$$
g(x)=\frac{-x\left(\alpha+\beta x^{p}\right)^{\nu} B\left(\frac{\mu+1}{p}, 1\right){ }_{3} F_{2}\left(\nu, \frac{\mu}{p}, \frac{\mu+1}{p} ; \frac{\mu}{p}+1, \frac{\mu+1}{p}+1 ; \frac{-\beta}{\alpha} x^{p}\right)}{\mu \alpha^{\nu}},
$$


if and only if $X$ has the distribution with the pdf (1), where $B\left(\frac{\mu+1}{p}, 1\right)$ represents the beta function and ${ }_{3} F_{2}\left(\nu, \frac{\mu}{p}, \frac{\mu+1}{p} ; \frac{\mu}{p}+1, \frac{\mu+1}{p}+1 ; \frac{-\beta}{\alpha} x^{p}\right)$ represents the generalized hypergeometric function (see, for example, Abramowitz and Stegun [8], Gradshteyn and Ryzhik [9], and Prudnikov et al. [10], among others).

Proof: Suppose that $E(X \mid X \leq x)=g(x) \frac{f(x)}{F(x)}$. Then, since $E(X \mid X \leq x)=\frac{\int_{0}^{x} u f(u) d u}{F(x)}$, we have $g(x)=\frac{\int_{0}^{x} u f(u) d u}{f(x)}$. Now, if the random variable $X$ satisfies the Assumption 2.1.1 and has the distribution with the pdf (1), then we have

$$
\begin{aligned}
& g(x)=\frac{\int_{0}^{x} u f(u) d u}{f(x)}=\frac{-\left.u(1-F(u))\right|_{0} ^{x}}{f(x)}+\frac{\int_{0}^{x}(1-F(u)) d u}{f(x)} \\
& =\frac{-x}{f(x)}+\frac{\int_{0}^{x}\left[1-\frac{\frac{p}{\mu}\left(\frac{\beta}{\alpha}\right)^{\frac{\mu}{p}} u^{\mu}}{\mathrm{B}\left(\frac{\mu}{p}, \nu-\frac{\mu}{p}\right)^{2}} F_{1}\left(\nu, \frac{\mu}{p} ; \frac{\mu}{p}+1 ; \frac{-\beta}{\alpha} u^{p}\right)\right] d u}{f(x)} \\
& =\frac{-\left(\alpha+\beta x^{p}\right)^{\nu} \int_{0}^{x}\left[u^{\mu}{ }_{2} F_{1}\left(\nu, \frac{\mu}{p} ; \frac{\mu}{p}+1 ; \frac{-\beta}{\alpha} u^{p}\right)\right] d u}{\mu \alpha^{\nu} x^{\mu-1}} \\
& =\frac{-x\left(\alpha+\beta x^{p}\right)^{\nu} B\left(\frac{\mu+1}{p}, 1\right){ }_{3} F_{2}\left(\nu, \frac{\mu}{p}, \frac{\mu+1}{p} ; \frac{\mu}{p}+1, \frac{\mu+1}{p}+1 ; \frac{-\beta}{\alpha} x^{p}\right)}{\mu \alpha^{\nu}},
\end{aligned}
$$

which follows from Prudnikov et al., [10], Vol. 3, Eq. 2.21.1.4, Page 314.

Conversely, suppose that

$$
g(x)=\frac{-x\left(\alpha+\beta x^{p}\right)^{\nu} B\left(\frac{\mu+1}{p}, 1\right){ }_{3} F_{2}\left(\nu, \frac{\mu}{p}, \frac{\mu+1}{p} ; \frac{\mu}{p}+1, \frac{\mu+1}{p}+1 ; \frac{-\beta}{\alpha} x^{p}\right)}{\mu \alpha^{\nu}} .
$$

Then, differentiating $g(x)$ with respect to $x$, using Lemma 2.1.1, and simplifying, we have

$$
g^{\prime}(x)=x-g(x)\left(\frac{\mu-1}{x}-\frac{\beta p x^{p-1}}{\alpha+\beta x^{p}}\right),
$$

from which we obtain

$$
\frac{x-g^{\prime}(x)}{g(x)}=\frac{\mu-1}{x}-\frac{\beta p x^{p-1}}{\alpha+\beta x^{p}} .
$$

Since, by Lemma 2.1.1, we have

$$
\frac{x-g^{\prime}(x)}{g(x)}=\frac{f^{\prime}(x)}{f(x)},
$$

it follows that

$$
\frac{f^{\prime}(x)}{f(x)}=\frac{\mu-1}{x}-\frac{\beta \nu p x^{p-1}}{\alpha+\beta x^{p}} .
$$

On integrating the above expression with respect to $x$ and simplifying, we obtain

$$
\ln f(x)=\ln \left(\frac{c x^{\mu-1}}{\left(\alpha+\beta x^{p}\right)^{\nu}}\right),
$$

or,

$$
f(x)=\frac{c x^{\mu-1}}{\left(\alpha+\beta x^{p}\right)^{\nu}},
$$


where $c$ is the normalizing constant to be determined. Thus, on integrating the above equation with respect to $x$ from $x=0$ to $x=\infty$, using the condition $\int_{0}^{\infty} f(x) d x=1$, and following Gradshteyn and Ryzhik [9], Equation 3.194.3, Page 285, we easily obtain

$$
c=\frac{p \alpha^{\nu}}{\operatorname{B}\left(\frac{\mu}{p}, \nu-\frac{\mu}{p}\right)\left(\frac{\beta}{\alpha}\right)^{-\frac{\mu}{p}}}
$$

and, hence, we have

$$
f_{X}(x)=\frac{p(\alpha)^{\nu-\frac{\mu}{p}}(\beta)^{\frac{\mu}{p}} x^{\mu-1}}{\mathrm{~B}\left(\frac{\mu}{p}, \nu-\frac{\mu}{p}\right)\left(\alpha+\beta x^{p}\right)^{\nu}},
$$

which is the required pdf (1). This completes the proof of Theorem 2.2.1.

Theorem 2.2.2. If the random variable $X$ satisfies the Assumption 2.1.1, with $\gamma=0$ and $\delta=\infty$, then $E(X \mid X \geq x)=\tilde{g}(x) \frac{f(x)}{1-F(x)}$, where

$$
\tilde{g}(x)=\frac{\int_{x}^{\infty} u f(u) d u}{f(x)}=\frac{E(X)}{f(x)}+\frac{\int_{0}^{x} u f(u) d u}{f(x)}=\frac{E(X)}{f(x)}+\frac{g(x)}{f(x)},
$$

if and only if $X$ has the distribution with the pdf (1), where $E(X)$, the first moment, is given by $(7), g(x)$ is given by (10), and $f(x)$ represents the pdf (1)

Proof: The proof is omitted, since it is similar to the Theorem 2.2.1, and easily follows from Lemma 2.1.2.

\subsection{Characterization by Order Statistics}

Suppose that $X_{1}, X_{2}, \ldots, X_{n}$ are $n$ independent random variables with the continuous cumulative distribution function $F(x)$ and probability density function $f(x)$. We assume that $X_{1, n} \leq X_{2, n} \leq \ldots \leq X_{n, n}$ are the corresponding order statistics. Then $X_{j, n} \mid X_{k, n}=x$, for $1 \leq k<j \leq n$, is distributed as the $(j-k)$ th order statistics from $(n-k)$ independent observations from the random variable $V$, and has the probability density function $f_{V}(v \mid x)$, where $f_{V}(v \mid x)=\frac{f(v)}{1-F(x)}, 0 \leq v<x$, see, for example, Ahsanullah et al. [11], chapter 5, or Arnold et al. [12], chapter 2. Further, $X_{i, n} \mid X_{k, n}=x, 1 \leq i<k \leq n$, is distributed as ith order statistics from $k$ independent observations from the random variable $W$ having the pdf $f_{W}(w \mid x)$ where $f_{W}(w \mid x)=\frac{f(w)}{F(x)}, w<x$. Let $S_{k-1}=\frac{1}{k-1}\left(X_{1, n}+X_{2, n}+\ldots+X_{k-1, n}\right)$, and $T_{k, n}=$ $\frac{1}{n-k}\left(X_{k+1, n}+X_{k+2, n}+\ldots+X_{n, n}\right)$.

Now, based on the order statistics, we will provide the characterizations in Theorem 2.3.1 and 2.3.2 below.

Theorem 2.3.1. Assume that the random variable $X$ satisfies the Assumption 2.1.1, with $\gamma=0$ and $\delta=\infty$, then $E\left(S_{k-1} \mid X_{k, n}=x\right)=$ $g(x) \tau(x)$, where $\tau(x)=\frac{f(x)}{F(x)}$, and

$$
g(x)=\frac{-x\left(\alpha+\beta x^{p}\right)^{\nu} B\left(\frac{\mu+1}{p}, 1\right){ }_{3} F_{2}\left(\nu, \frac{\mu}{p}, \frac{\mu+1}{p} ; \frac{\mu}{p}+1, \frac{\mu+1}{p}+1 ; \frac{-\beta}{\alpha} x^{p}\right)}{\mu \alpha^{\nu}},
$$

if and only if $f_{X}(x)=\frac{\frac{\mu}{p}(\alpha)^{\nu-} \frac{\mu}{p}(\beta)^{\frac{\mu}{p}} x^{\mu-1}}{\mathrm{~B}\left(\frac{\mu}{p}, \nu-\frac{\mu}{p}\right)\left(\alpha+\beta x^{p}\right)^{\nu}}$.

Proof: Since $E\left(S_{k-1} \mid X_{k, n}=x\right)=E(X \mid X \leq x)$, see David and Nagaraja [13], the proof of Theorem 2.3.1 easily follows from Theorem 2.2.1.

Theorem 2.3.2. Assume that the random variable $X$ satisfies the Assumption 2.1.1, with $\gamma=0$ and $\delta=\infty$. Then, $E\left(T_{k, n} \mid X_{k, n}=x\right)=$ $\tilde{g}(x) r(x)$, where $r(x)=\frac{f(x)}{1-F(x)}$ and

$$
\tilde{g}(x)=\frac{\int_{x}^{\infty} u f(u) d u}{f(x)}=\frac{E(X)}{f(x)}+\frac{\int_{0}^{x} u f(u) d u}{f(x)}=\frac{E(X)}{f(x)}+\frac{g(x)}{f(x)},
$$


if and only if $f_{X}(x)=\frac{p(\alpha)^{\nu-\frac{\mu}{p}}(\beta)^{\frac{\mu}{p}} x^{\mu-1}}{\mathrm{~B}\left(\frac{\mu}{p}, \nu-\frac{\mu}{p}\right)\left(\alpha+\beta x^{p}\right)^{\nu}}$.

Proof: Since $E\left(T_{k, n} \mid X_{k, n}=x\right)=E(X \mid X \geq x)$, see David and Nagaraja [13], the proof of Theorem 2.3.2 easily follows from Theorem 2.2.2.

\subsection{Characterization by Upper Record Values}

Suppose that $X_{1}, X_{2}, \ldots$, is a sequence of independent and identically distributed absolutely continuous random variables with cumulative distribution function $F(x)$ and probability density function $f(x)$. Let $Y_{n}=\max \left(X_{1}, X_{2}, \ldots, X_{n}\right)$ for $n \geq 1$. We say that $X_{j}$ is an upper record value of $\left\{X_{n}, n \geq 1\right\}$ if $Y_{j}>Y_{j-1}, j>1$. The indices at which the upper records occur are given by the record times $\left\{U(n)>\min \left(j \mid j>U(n+1), X_{j}>X_{U(n-1)}, n>1\right)\right\}$ and $U(1)=1$. We will denote the $n$th upper record value as $X(n)=X_{U(n)}$. For details on record values, see Ahsanullah and Nevzorov [14], among others. In the following theorem, we will provide the characterization based on upper record values.

Theorem 2.4.1. Assume that the random variable $X$ satisfies the Assumption 2.1.1, with $\gamma=0$ and $\delta=\infty$. Then, $E(X(n+1) \mid X(n)=x)=$ $\tilde{g}(x) r(x)$, where $r(x)=\frac{f(x)}{1-F(x)}$ and

$$
\tilde{g}(x)=\frac{\int_{x}^{\infty} u f(u) d u}{f(x)}=\frac{E(X)}{f(x)}+\frac{\int_{0}^{x} u f(u) d u}{f(x)}=\frac{E(X)}{f(x)}+\frac{g(x)}{f(x)}
$$

if and only if

$$
f_{X}(x)=\frac{p(\alpha)^{\nu-\frac{\mu}{p}}(\beta)^{\frac{\mu}{p}} x^{\mu-1}}{B\left(\frac{\mu}{p}, \nu-\frac{\mu}{p}\right)\left(\alpha+\beta x^{p}\right)^{\nu}} .
$$

Proof: Since $E(X(n+1) \mid X(n)=x)=E(X \mid X \geq x)$, see Nevzorov [15], the proof of Theorem 2.4.1 easily follows from Theorem 2.2.2.

\section{RELIABILITY ANALYSIS}

The reliability analysis of lifetime distributions plays an important role in modelling many real world phenomena in the fields of biological, economics, engineering, physical, and other pure and applied sciences. For a nonrepairable population, we define the failure rate as the instantaneous rate of failure for the survivors to time $t$ during the next instant of time. We investigate some reliability properties of the said model. The corresponding survival (or reliability) and the hazard (or failure rate) functions of the said model are respectively given by

$$
S(x)=R(x)=1-F_{X}(x)=1-\frac{\frac{p}{\mu}\left(\frac{\beta}{\alpha}\right)^{\frac{\mu}{p}} x^{\mu}}{\mathrm{B}\left(\frac{\mu}{p}, \nu-\frac{\mu}{p}\right)}{ }_{2} F_{1}\left(\nu, \frac{\mu}{p} ; \frac{\mu}{p}+1 ; \frac{-\beta}{\alpha} x^{p}\right),
$$

and

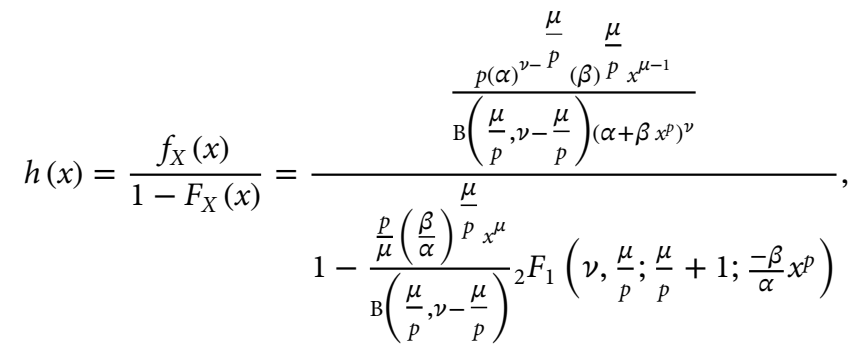

where $x>0, \alpha>0, \beta>0, \mu>0, \nu>0, p>0$, and $\nu p>\mu$. Differentiating (12) with respect to $x$, we have

$$
\begin{aligned}
h^{\prime}(x) & =\frac{f^{\prime}(x)}{f(x)} h(x)+[h(x)]^{2} \\
& =\frac{\left(\alpha(\mu-1)-\beta(\nu p-\mu+1) x^{p}\right) h(x)}{x\left(\beta x^{p}+\alpha\right)}+[h(x)]^{2},
\end{aligned}
$$


for $x>0$. In order to discuss the behavior of the failure rate function, $h(x)$, let $h^{\prime}(x)=0$. We observe that the nonlinear equation $h^{\prime}(x)=0$ does not have a closed form solution, but could be solved numerically by using some mathematical software such as Maple, Mathematica, and R. It is obvious from (13) that $h^{\prime}(x)$ is positive irrespective of the values of the parameters. This confirms the Increasing Failure Rate (IFR) property of said model. For some special values of the parameters, the graphs of the hazard function (12) are illustrated in Fig. 2 (a and b) below.

The effects of the parameters are obvious from these figures. The increasing, then decreasing, and upside-down bathtub shape behaviors of the failure rate function, $h(x)$, are observed from these figures. Further, it is also sometimes useful to find the average failure rate function $(A F R)$, over any interval, say, $(0, t)$, that averages the failure rate over the interval, $(0, t)$, see, for example, Barlow and Proschan [16]. Thus, the AFR) of the distribution of the said model, over the interval $(0, t)$, is given by

$$
A F R=\frac{-\ln (R(t))}{t}=-\frac{1}{t} \ln \left(1-\frac{\frac{p}{\mu}\left(\frac{\beta}{\alpha}\right)^{\frac{\mu}{p}} t^{\mu}}{\mathrm{B}\left(\frac{\mu}{p}, \nu-\frac{\mu}{p}\right)}{ }_{2} F_{1}\left(\nu, \frac{\mu}{p} ; \frac{\mu}{p}+1 ; \frac{-\beta}{\alpha} t^{p}\right)\right)
$$

which in view of the expansion of logarithmic function as a power series, is seen to be positive irrespective of the values of the parameters, and hence the distribution of the said model is increasing failure rate on average, that is, Increasing Failure Rate on Average (IFRA). Also, recall that a life distribution $F$ (.) is NBU (new better than used) if $R(x+y) \leq R(x) R(y), \forall x, y \geq 0$, and NWU (new worse than used) if the reversed inequality holds, see, for example, Barlow and Proschan [16]. We note that, for the said model, since

$$
R(x+y)=1-\frac{\frac{p}{\mu}\left(\frac{\beta}{\alpha}\right)^{\frac{\mu}{p}}(x+y)^{\mu}}{\mathrm{B}\left(\frac{\mu}{p}, \nu-\frac{\mu}{p}\right)} F_{1}\left(\nu, \frac{\mu}{p} ; \frac{\mu}{p}+1 ; \frac{-\beta}{\alpha}(x+y)^{p}\right),
$$

and

$$
\begin{aligned}
& R(x) . R(y) \\
& =\left\{1-\frac{\frac{p}{\mu}\left(\frac{\beta}{\alpha}\right)^{\frac{\mu}{p}}(x)^{\mu}}{\mathrm{B}\left(\frac{\mu}{p}, \nu-\frac{\mu}{p}\right)}{ }_{2} F_{1}\left(\nu, \frac{\mu}{p} ; \frac{\mu}{p}+1 ; \frac{-\beta}{\alpha}(x)^{p}\right)\right\} \\
& \times\left\{1-\frac{\frac{p}{\mu}\left(\frac{\beta}{\alpha}\right)^{\frac{\mu}{p}}(y)^{\mu}}{\mathrm{B}\left(\frac{\mu}{p}, \nu-\frac{\mu}{p}\right)^{2}} F_{1}\left(\nu, \frac{\mu}{p} ; \frac{\mu}{p}+1 ; \frac{-\beta}{\alpha}(y)^{p}\right)\right\},
\end{aligned}
$$

it is easily seen that $R(x+y) \leq R(x) . R(y)$, which implies that the distribution of the said model has the property of NBU.

(a)

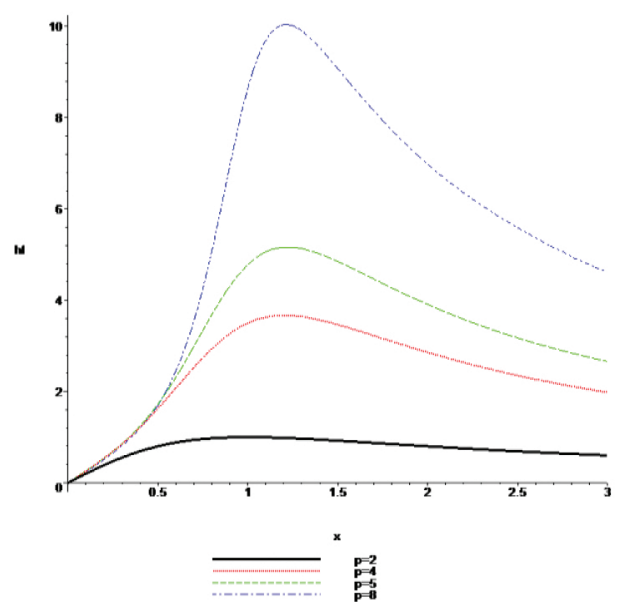

(b)

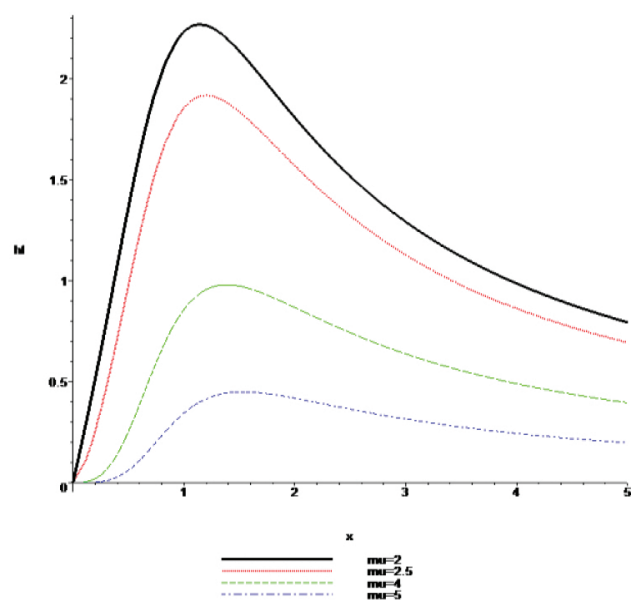

Figure 2 Failure Rate (hf) Plots for (a) $\alpha=1, \beta=1, v=2, \mu=2, p=2,4,5,8$ (left); and (b) $\alpha=1, \beta=1, v=2$, $p=3, \mu=2,2.5,4,5$ (right). 


\section{ESTIMATION, SIMULATION, AND AIC OF THE PERFORMANCE OF GENERALIZED BURR LIFE-TESTING MODEL}

\subsection{The Method of Moments}

From the $k t h$ moment, $E\left(X^{k}\right)$, in (6) of the said model, taking $k=1,2,3,4,5$, and evaluating the respective expressions numerically, we obtain the first five moments. Then, the moment estimations (MME's) of the parameters $\alpha>0, \beta>0, \mu>0, \nu>0$, and $p>0$ can be determined by solving the system of five equations obtained from (6) by Newton-Raphson's iteration method, and using the computer package such as Maple, Mathematica 9, or R, MathCAD14, or other software.

\subsection{The Method of Maximum Likelihood}

Given a sample $\left\{x_{i}\right\}, i=1,2,3, \ldots, n$, the likelihood function of $(1)$ is given by $L=\prod_{i=1}^{n} f\left(x_{i}\right)$. The objective of the likelihood function approach is to determine those values of the parameters that maximize the function $L$. Suppose that $R=\ln (L)=\sum_{i=1}^{n} \ln \left[f\left(x_{i}\right)\right]$. Then, upon differentiation, the maximum likelihood estimates (MLE) of the parameters $\alpha>0, \beta>0, \mu>0, \nu>0$, and $p>0$ are obtained by solving the maximum likelihood equations $\frac{\partial R}{\partial \alpha}=0, \frac{\partial R}{\partial \beta}=0, \frac{\partial R}{\partial \mu}=0, \frac{\partial R}{\partial \nu}=0$, and $\frac{\partial R}{\partial p}$, applying the Newton-Raphson's iteration method, and using the computer package such as Maple, Mathematica 9, or R, MathCAD14, or other software.

\subsection{Simulation}

In this section, we use simulation to compare the performances of the different methods of estimation mainly with respect to their biases, mean square errors (MSE), and variances for different sample sizes. A numerical study is performed using Mathematica 9 software. Different sample sizes are considered through the experiments at size $n=15,20,30$, and 50 for different values of the parameters $\alpha>0, \beta>0, \mu>$ $0, \nu>0$, and $p>0$. The experiment is repeated 1000 times. In each experiment, the estimates of the parameters are obtained by the method of MLE. The means, MSEs and biases for the different estimators are reported from these experiments in Table 1 respectively below.

If we review Table 1, it is observed that as the sample size increases, both absolute bias and MSE decrease and converge to close to true values of parameters.

Table 1 The parameter estimation of the generalized burr life-testing model using MLE.

\begin{tabular}{|c|c|c|c|c|c|c|c|c|c|}
\hline$n$ & Par & Init & MLE & Bais & MSE & Init & MLE & Bais & MSE \\
\hline \multirow{5}{*}{10} & $\alpha$ & 1.2 & 1.2536 & 0.0535 & 0.0219 & 1.4 & 1.4435 & 0.0435 & 0.0240 \\
\hline & $\beta$ & 1.2 & 1.1700 & -0.0300 & 0.0195 & 1.4 & 1.3829 & -0.0171 & 0.0252 \\
\hline & $v$ & 1.4 & 1.0709 & -0.3291 & 0.1736 & 1.6 & 1.2408 & -0.3593 & 0.3325 \\
\hline & $\mu$ & 0.5 & 0.4269 & -0.0731 & 0.0085 & 0.5 & 0.4348 & -0.0652 & 0.0084 \\
\hline & $\mathrm{p}$ & 0.8 & 1.5221 & 0.7221 & 0.9093 & 0.7 & 0.8870 & 0.1870 & 0.0993 \\
\hline \multirow{5}{*}{20} & $\alpha$ & 1.2 & 1.2437 & 0.0437 & 0.0101 & 1.4 & 1.4271 & 0.0271 & 0.0104 \\
\hline & $\beta$ & 1.2 & 1.1644 & -0.0356 & 0.0102 & 1.4 & 1.3842 & -0.0158 & 0.0128 \\
\hline & $\nu$ & 1.4 & 1.0480 & -0.3520 & 0.1424 & 1.6 & 1.1871 & -0.4129 & 0.1937 \\
\hline & $\mu$ & 0.5 & 0.4240 & -0.0760 & 0.0073 & 0.5 & 0.4344 & -0.0656 & 0.0064 \\
\hline & $\mathrm{p}$ & 0.8 & 1.4138 & 0.6138 & 0.5405 & 0.7 & 0.8703 & 0.1703 & 0.0618 \\
\hline \multirow{5}{*}{30} & $\alpha$ & 1.2 & 1.2390 & 0.0390 & 0.0069 & 1.4 & 1.4216 & 0.0215 & 0.0069 \\
\hline & $\beta$ & 1.2 & 1.1649 & -0.0351 & 0.0075 & 1.4 & 1.3854 & -0.0146 & 0.0087 \\
\hline & $\nu$ & 1.4 & 1.0382 & -0.3618 & 0.1413 & 1.6 & 1.1794 & -0.4206 & 0.1932 \\
\hline & $\mu$ & 0.5 & 0.4241 & -0.0759 & 0.0068 & 0.5 & 0.4343 & -0.0657 & 0.0057 \\
\hline & $\mathrm{p}$ & 0.8 & 1.3944 & 0.5944 & 0.4625 & 0.7 & 0.8622 & 0.1622 & 0.0488 \\
\hline \multirow{5}{*}{50} & $\alpha$ & 1.2 & 1.2386 & 0.0385 & 0.0044 & 1.4 & 1.4273 & 0.0273 & 0.0047 \\
\hline & $\beta$ & 1.2 & 1.1608 & -0.0392 & 0.0049 & 1.4 & 1.3743 & -0.0257 & 0.0057 \\
\hline & $v$ & 1.4 & 1.0354 & -0.3646 & 0.1382 & 1.6 & 1.1835 & -0.4165 & 0.1826 \\
\hline & $\mu$ & 0.5 & 0.4223 & -0.0777 & 0.0066 & 0.5 & 0.4297 & -0.0703 & 0.0058 \\
\hline & $\mathrm{p}$ & 0.8 & 1.3558 & 0.5558 & 0.3626 & 0.7 & 0.8410 & 0.1410 & 0.0331 \\
\hline
\end{tabular}

MLE, maximum likelihood estimate; MSE, mean square errors. 


\subsection{Akaike Information Criterion}

As pointed out by Emiliano et al. [17], the choice of the best model is crucial in modeling data. Akaike [18,19] developed an entropy-based information criterion to test the applicability, relative measures, and performance of lifetime distributions to real life data, known in the literature as the AIC. Later, various information criteria for testing the goodness of fit of lifetime probability models were developed by different authors and researchers, among them the corrected Akaike information criterion (CAIC) (Sugiura [20]), the Bayesian information criterion (BIC) (Schwarz [21]), the generalized information criterion (GIC) (Konishi and Kitagawa [22]), and Hannan-Quinn information criterion (HQIC) (Hannan and Quinn [23]), are notable. For details on these, the interested readers are referred to Emiliano et al. [17], and references therein. In this section, we investigate the performance of the generalized Burr life-testing model by using various criteria such as $-2 \ln L, A I C, B I C$, the CAIC, and the HQIC. For this, we consider some health related data as described in Example 1 below.

\section{Example 1.}

This example considers the data for average annual percent change in private health insurance premiums (All Benefits: Health Services and Supplies), Calendar Years 1969-2007 (SOURCE: Centers for Medicare \& Medicaid Services, Office of the Actuary, National Health Statistics Group), which are provided in Table 2. Based on this example, we test the goodness of fit of the generalized Burr life-testing model and compare it with the Burr's type XII, log-logistic, gamma, and lognormal distributions.

The mean, median, skewness, and kurtosis of this data are 10.7, 10.1, 0.603, and 0.557 respectively. We can see that the data is right skewed. The estimators of the unknown parameters of each distribution is obtained by the maximum likelihood method. In order to compare the generalized Burr life-testing model with other models, we use $-2 \ln L$ critrion, AIC, BIC, the correct Akaike information criterion (CAIC), Hannan information criterion $(H Q I C)$, which are defined as follows:

$$
\begin{gathered}
A I C=2 k-2 \ln L, \quad C A I C=A I C+\frac{2 k(k+1)}{n-k-1}, \\
B I C=k \ln (n)-2 \ln L, \quad \text { and } \quad H Q I C=2 k \ln [\ln (n)]-2 \ln L,
\end{gathered}
$$

where $k$ is the number of parameters in the statistical model, $n$ is the sample size, and $\ln L$ is the maximized value of the log-likelihood function under the considered model. The "best" distribution corresponds to the smallest values of $-2 \ln L, A I C, B I C, C A I C$, and HQIC. Table 3 shows the MLEs of the model parameters and its standard error (S.E) (in parentheses) for the data set in Table 2.

The variance covariance matrix $I(\hat{\lambda})^{-1}$ of the MLEs of the generalized Burr life-testing model, with the pdf (1), is computed for the data set in Table 2 as follows:

$$
\left(\begin{array}{rlrrc}
3.698 & 0.035 & 183.332 & -49.764 & -12.657 \\
0.035 & 2.928 \times 10^{-3} & 0.079 & -0.022 & -5.476 \times 10^{-3} \\
183.332 & 0.079 & 127.361 & -94.167 & -6.765 \\
-49.764 & -0.022 & -94.167 & 107.371 & 6.125 \\
-12.657 & -5.476 \times 10^{-3} & -6.765 & 6.125 & 0.393
\end{array}\right) .
$$

The following Table 4 shows the values of AIC, BIC,CAIC, and HQIC statistics for the data set in Table 2.

In Fig. 3 below, we provide the plots of estimated densities of the fitted generalized Burr life-testing model, Burr's type XII, log-logistic, gamma and lognormal models for the data set in Table 2.

In our above analysis, the Table 3 shows the MLE's of the parameters of the fitted generalized Burr life testing and other distributions to the data set in Table 2. Further, Table 4 shows the values of AIC, BIC,CAIC, and HQIC. The values in Tables 3 and 4 indicate that the generalized Burr life-testing distribution is a strong competitor to other distributions used here for fitting to the data set in Table 2 . A density plot in

Table 2 Average annual percent change in private health insurance premiums.

$14.4,14.0,15.4,9.4,11.7,15.0,24.9,20.7,12.5,14.9,12.6,16.7,13.8,11.0,12.9,10.1,1.9,8.5,16.5,15.3,13.3,9.8,8.4$
$7.9,3.7,5.1,4.6,4.4,5.4,6.1,8.0,10.0,11.2,10.1,6.4,6.7,5.7,5.8$

Table 3 The MLEs and S.E of the model parameters for data set in Table 2.

\begin{tabular}{lcccc}
\hline \multicolumn{4}{c}{ Model } & \multicolumn{3}{c}{ MLEs and S. E } \\
\hline New Model $(\alpha, \beta, \nu, \mu, p)$ & $3.291(1.92311)$ & $1.561 \times 10^{-3}\left(5.411 \times 10^{-3}\right)$ & $15.238(11.285)$ & $16.571(10.362) 2.332(0.627)$ \\
Burr XII $(a, b)$ & $0.063(0.02923)$ & $4.916(1.945)$ & - & - \\
Gamma $(\theta, \gamma)$ & $0.776(0.17506)$ & $19.969(2.2)$ & - & - \\
Log Logistic $(\lambda, k)$ & $0.04(0.0081)$ & $7.851(0.073)$ & - & - \\
Log normal $(\eta, \sigma)$ & $3.223(0.03234)$ & $0.22\left(6.77 \times 10^{-3}\right)$ & - & - \\
\hline
\end{tabular}


Table 4 The AIC, BIC, CAIC, and HQIC statistics for the data set in Table 2.

\begin{tabular}{lccccc}
\hline Distribution & -2ln $\boldsymbol{L}$ & AIC & BIC & CAIC & HQIC \\
\hline New Model & 250.804 & 260.804 & 258.814 & 262.569 & 263.857 \\
Burr XII & 431.625 & 435.625 & 434.829 & 435.949 & 436.846 \\
Gamma & 504.553 & 508.553 & 507.757 & 508.877 & 509.774 \\
Log Logistic & 682.54 & 439.075 & 438.279 & 439.4 & 440.297 \\
Log normal & 473.113 & 477.113 & 476.317 & 477.437 & 478.334 \\
\hline
\end{tabular}

AIC, Akaike information criterion; BIC, Bayesian information criterion; CAIC, corrected Akaike information criterion; HQIC, Hannan-Quinn information criterion.

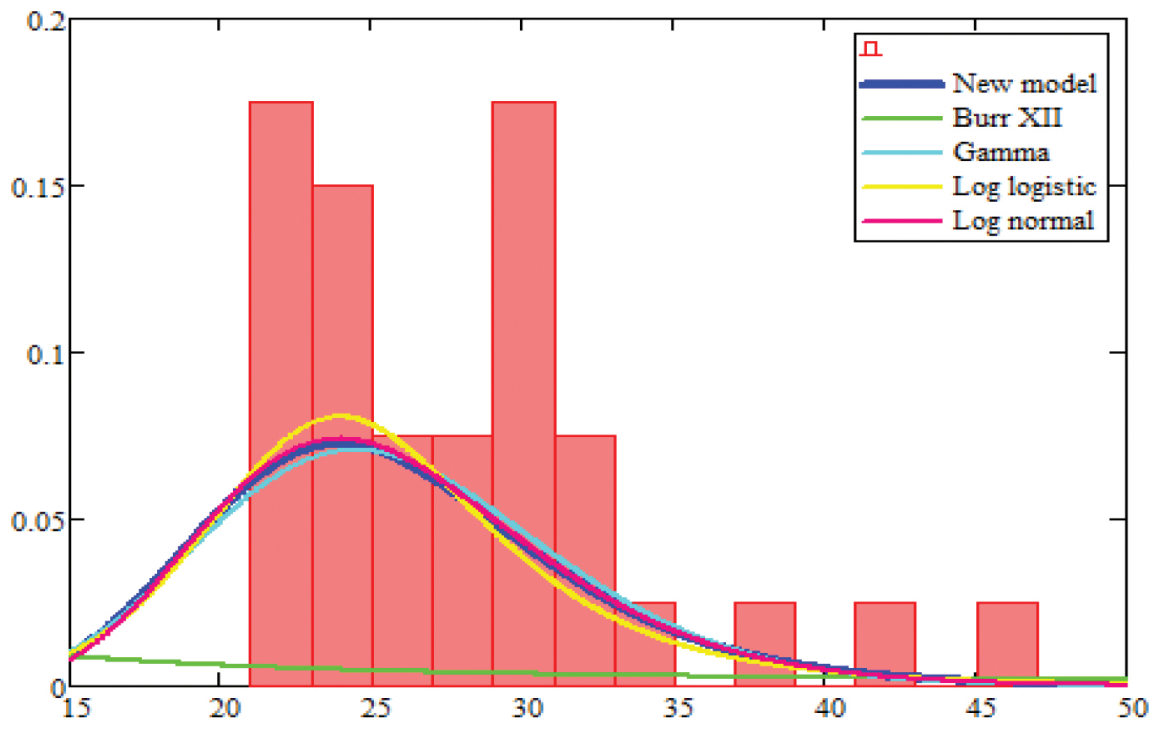

Figure 3 Estimated densities of the models for data set in Table 2.

Fig. 3 compares the fitted densities of the models with the empirical histogram of the observed data. The fitted density for generalized Burr life-testing distribution is the closest to the empirical histogram of the other models.

\section{CONCLUDING REMARKS}

Since the truncated distributions arise in practical statistics where the ability of record observations is limited to a given threshold or within a specified range, we investigate, in this paper, some characterizations of the five-parameter continuous life-testing continuous probability distribution of Shakil and Kibria [1] by truncated first moment, order statistics, and upper record values. We also investigate some reliability properties, and Akaike $(A I C)$ and other information criterion to test the measure of the goodness of fit of the said model. It is hoped that the findings of this paper will be quite useful for researchers in the fields of reliability, probability, statistics, and other applied sciences.

\section{CONFLICT OF INTEREST}

The authors declare that they have no Conflict of Interests or Competing Interests.

\section{AUTHORS' CONTRIBUTIONS}

All the authors have fully contributed to the article, and have read and approved the final manuscript.

\section{Funding Statement}

There are no sources of funding for the research. 


\section{ACKNOWLEDGMENT}

The authors are thankful to the editor-in-chief and the referees for their valuable comments and suggestions, which certainly improved the presentation of the paper.

\section{REFERENCES}

1. M. Shakil, B.M.G. Kibria, J. Stat. Theory Appl. 9 (2010), 255-282.

2. J. Galambos, S. Kotz, Characterizations of Probability Distributions. A Unified Approach with an Emphasis on Exponential and Related Models. Lecture Notes in Mathematics, vol. 675, Springer, Berlin, 1978.

3. S. Kotz, D.N. Shanbhag, Adv. Appl. Probab. 12 (1980), 903-921.

4. W. Glänzel, A. Telcs, A. Schubert, Z. Wahrsch. Verw. Gebiete. 66 (1984), 173-183.

5. W. Glänzel, in: P. Bauer, F. Konecny, W. Wertz, Mathematical Statistics and Probability Theory, vol. B, Reidel, Dordrecht, 1987, pp. 75-84.

6. M. Ahsanullah, Characterizations of Univariate Continuous Distributions, Atlantis-Press, Paris, 2017.

7. M. Ahsanullah, M. Shakil, Int. J. Adv. Stat. Probab. 6 (2018), 42-49.

8. M. Abramowitz, I.A. Stegun, Handbook of Mathematical Functions with Formulas, Graphs, and Mathematical Tables, National Bureau of Standards, Washington, 1970.

9. I.S. Gradshteyn, I.M. Ryzhik, Table of Integrals, Series, and Products, Academic Press, Inc., San Diego, 2000.

10. A.P. Prudnikov, U.A. Brychkov, O.I. Marichev, Integrals and Series, vol. 3, Gordon and Breach Science Publishers, Amsterdam, 1986.

11. M. Ahsanullah, V.B. Nevzorov, M. Shakil, An Introduction to Order Statistics, Atlantis-Press, Paris, 2013.

12. B.C. Arnold, N. Balakrishnan, H.N. Nagaraja, First Course in Order Statistics, Wiley, New York, 2005.

13. H.A. David, H.N. Nagaraja, Order Statistics, third ed., Wiley, New York, 2003.

14. M. Ahsanullah, V.B. Nevzorov, Records via Probability Theory, Atlantis-Press, Paris, 2015.

15. V.B. Nevzorov, Records: Mathematical Theory, Translation of Mathematical Monograph, American Mathematical Society, Rhode Island, 2001.

16. R.E. Barlow, F. Proschan, Mathematical Theory of Reliability, Wiley, New York, 1965.

17. P.C. Emiliano, M.J. Vivanco, F.S. De Menezes, Comput. Stat. Data Anal. 69 (2014), 141-153.

18. H. Akaike, in: B.N. Petrov, F. Csaki, Second International Symposium on Information Theory, Akademiai Kiado, Budapest, 1973, pp. $267-281$.

19. H. Akaike, IEEE Trans. Autom. Control. 19 (1974), 717-723.

20. N. Sugiura, Commun. Stat. Theory Methods. 7 (1978), 13-26.

21. G. Schwarz, Ann. Stat. 6 (1978), 461-464.

22. S. Konishi, G. Kitagawa, Information Criteria and Statistical Modeling, Springer, New York, 2008.

23. E.J. Hannan, B.G. Quinn, J. Royal Stat. Soc. Ser. B. 41 (1979), 190-195. 